\title{
Early outcome and predictors of early outcome in patients treated surgically for central cord syndrome
}

\author{
Christopher K. Kepler, MD, MBA, ${ }^{1}$ Christopher Kong, MD, ${ }^{2}$ Gregory D. Schroeder, MD, ${ }^{1}$ \\ Nikolaus Hjelm, MD, ${ }^{3}$ Amir Sayadipour, MD, ${ }^{3}$ Alexander R. Vaccaro, MD, PhD, and \\ D. Greg Anderson, MD'
}

${ }^{1}$ Rothman Institute at ${ }^{3}$ Thomas Jefferson University, Philadelphia, Pennsylvania; and ${ }^{2}$ University of British Columbia, Vancouver, British Columbia, Canada

OBJECT The purpose of this study was to characterize changes in American Spinal Injury Association motor score (AMS) in the 1st week after traumatic central cord syndrome (CCS) to identify predictors of improved early outcome in patients treated with early versus delayed surgical intervention.

METHODS All patients presenting to a regional spinal cord injury center between January 2004 and June 2009 were queried for those with a diagnosis of CCS. Patients treated conservatively were excluded. A prospectively maintained spinal cord injury database was used to track AMS throughout each patient's hospitalization. Hospital records provided information regarding demographics, presenting neurological examination, imaging findings, comorbidities, timing and nature of surgical procedures, and length of stay (LOS) in the hospital and intensive care unit (ICU). Patients were separated into those who underwent early surgery, within 1 day of presentation (early group), and those who underwent surgery on a delayed basis (delayed group). Differences between groups were analyzed using the Student t-test and chisquare test. Predictors of outcome were identified using correlation analysis and multiple linear regression.

RESULTS Of 426 patients in the database, 80 (18.8\%) were diagnosed as having CCS, and 68 of them ultimately underwent surgical decompression. Nineteen (28\%) of 68 patients underwent surgery within 1 day of presentation (early group) while the remaining 49 patients (72\%) underwent surgery on a delayed basis (delayed group). The mean age in the early group was significantly younger than that of the delayed group ( 52 vs 59 years, $p=0.049$ ). Other characteristics were similar between groups including sex, proportion of patients with cord edema on MRI (44\% early vs 55\% delayed, $p=0.47)$, and proportion of patients with cervical fracture $(26 \%$ early vs $28 \%$ delayed, $p=0.98)$. Patients in the early group presented with an AMS of 62.5 versus 70.0 for the delayed group ( $p=0.36)$. No difference in the change in AMS was seen at 7 days between the early group ( -2.9 points) and the delayed group ( -4.2 points) $(p=0.34)$. Additionally, the number of patients who had early improvement was similar between the early and delayed groups $(50 \%$ vs $48 \%$, respectively, $p=0.94)$. Neither time in the ICU (3.4 vs 3.4 days, $p=0.84$ ) nor the overall LOS (10.5 vs 12.5 days, $p=$ $0.59)$ was different in the early versus delayed groups, respectively. Correlation analysis and multiple linear regression demonstrated early surgery was not associated with change in AMS or AMS at Day 7. Age was identified as the only significant predictor of change in AMS and had a negative effect (coefficient $=-0.34, p=0.025$ ).

\begin{abstract}
ABBREVIATIONS AMS = ASIA motor score; ASIA = American Spinal Injury Association; CCS = central cord syndrome; ICU = intensive care unit; LOS = length of stay. SUBMITTED October 3, 2014. ACCEPTED January 16, 2015.

INCLUDE WHEN CITING Published online July 10, 2015; DOI: 10.3171/2015.1.SPINE141013.

DISCLOSURE Dr. Kepler reports being a board member/committee appointee for the Association of Collaborative Spine Research, a consultant for Healthgrades Inc., and receiving support from CSRS and NASS for non-study-related clinical or research efforts. Dr. Schroeder reports receiving financial support from Medtronic to attend SRS and CSRS annual meetings. Dr. Vaccaro reports receiving royalties from DePuy, Medtronic, Stryker Spine, Biomet Spine, Globus, and Aesculap; receiving royalties or financial support from the following publishers: Elsevier, Thieme, Jaypee, and Taylor and Francis; being a consultant for Gerson Lehrman Group, Guidepoint Global, Medacorp, Innovative Surgical Design, Expert Testimony, Ellipse, Globus, Stryker, Medtronic, Stout Medical, and Orthobullets; owning stock or stock options in Globus Medical, Progressive Spinal Technologies, Advanced Spinal Intellectual Properties, Computational Biodynamics, Stout Medical, Paradigm Spine, Replication Medica, Spinology, Spine Medica, Vertiflex, Small Bone Technologies, Crosscurrent, Syndicom, In Vivo, Flagship Surgical, Location Based Intelligence, Gamma Spine, Cytonics, Bonovo Orthopaedics, Electrocore, Flowpharma, RSI, Rothman Institute (RI) and related properties, Innovative Surgical Design, and Spinicity; receiving research support from Cerapedics and AO Spine; being on the editorial governing board for Spine, JNS: Spine, Pan Arab Journal of Neurosurgery, and European Spine Journal; being a board member or having a committee appointment for AO Spine, Innovative Surgical Design, Association of Collaborative Spine Research, Spinicity, Progressive Spinal Technologies, Computational Biodynamics, Advanced Spinal Intellectual Properties, Location Based Intelligence, RSI, and RI and related properties. Dr. Anderson reports receiving royalties from Medtronic and DePuy; being on the speakers bureau for DePuy and Medtronic; being a consultant for DePuy Synthes, Medtronic, and Globus Medical; owning stock in ISD, PST, and Spinicity; receiving research support from DePuy; receiving royalties from Thieme; being on the editorial board for Journal of Spine Disorders and Techniques, Spine Universe, and Spinal Deformity; and being a board member or committee appointee for CSRS and SMISS.
\end{abstract}


CONCLUSIONS Early treatment of patients with CCS remains controversial. Although some long-term neurological recovery is expected in patients with a CCS, surgeons and patients should not expect early neurological improvement with or without early operative intervention.

http://thejns.org/doi/abs/10.3171/2015.1.SPINE141013

KEY WORDS central cord syndrome; early decompression; delayed decompression; neurologic recovery; AMS; spinal cord injury

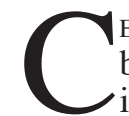
ENTRAL cord syndrome (CCS) was first described by Schneider in 1954 as an incomplete spinal cord injury causing tetraplegia with weakness more pronounced in the upper than the lower extremities. Some degree of bowel and bladder dysfunction and various patterns of sensory loss are not uncommon. ${ }^{16} \mathrm{CCS}$ is relatively well characterized as it is the most common of all incomplete spinal cord injury syndromes. ${ }^{2,13}$ Unfortunately, it also is the subject of controversy as there is lack of clarity in terms of optimal management strategy.

In his original description of CCS, Schneider reported that surgical management was contraindicated. This was due to a combination of poor surgical outcomes he witnessed following cord decompression and remarkable neurological recovery he observed in patients treated with medical management alone. ${ }^{16}$ Since 1954 , however, advances in spine surgery, intensive care, and anesthesia have mitigated surgical complications and improved surgical results. Today, surgical decompression with or without stabilization is considered standard of care for many incomplete spinal cord injuries presenting with ongoing cord compression. A systematic review by La Rosa and colleagues in 2004 showed that decompression of incomplete cord injuries within 24 hours was associated with superior neurological outcomes at 6-month follow-up or later. ${ }^{9}$ For CCS, however, timing of surgery has remained more controversial.

This controversy is partly due to the heterogeneity of injury mechanisms seen in patients with CCS. Some authors have thus subcategorized the syndrome to reflect this diversity in posttraumatic and predisposing anatomy. ${ }^{6,17}$ Harrop et al. (2006) conceptually divided patients with CCS into young patients $(<50$ years old) with focal instability, young patients with focal disc herniation causing local cord compression, and elderly patients $(>50$ years old) with spondylosis and no focal instability or fracture. ${ }^{6}$ The latter category, known as "spondylotic CCS," is the most common form of $\mathrm{CCS}^{8,16}$ and most commonly occurs as a result of low energy falls. While many patients with CCS may receive some form of early or delayed surgery, patients with spondylotic CCS are unique in that they may not undergo surgery, depending on the preferences of the treating surgeon.

Nonoperative management and close observation are commonly implemented for patients with spondylotic CCS. As significant, spontaneous neurological recovery is expected, decompressive surgery has not always been pursued, unless there is demonstrable functional decline or failure to improve. In an assessment of this management model, Lenehan et al. performed a prospective review of 73 patients who presented with spondylotic CCS. The authors found that patients who received decompression within 24 hours of injury demonstrated a significant improvement in American Spinal Injury Association (ASIA) motor scores (AMS) at both 6 and 12 months postinjury when compared with patients treated with either delayed decompression ( $>24$ hours) or nonoperative management. Combining their results with expert opinion, their group recommended that patients with spondylotic CCS should receive decompression within 24 hours if presenting as ASIA Grade $\mathrm{C}$ or worse and medical management with close observation if presenting as ASIA Grade D. ${ }^{10}$ To our knowledge, the effect of these recommendations on early neurological outcomes has not yet been clarified nor have their results been confirmed.

The purpose of this study was to compare changes in AMS between CCS patients receiving either early $(<24$ hours postinjury) or delayed ( $>24$ hours) surgical management and to characterize predictors of early outcome in the combined cohort. Severity of neurological improvement was assessed using the AMS instead of ASIA grade because AMS allows for greater sensitivity with separate scoring for each limb and has been found to be the best indicator for observing the effects of therapeutic interventions. ${ }^{5,12}$

\section{Methods}

The study was approved by the institutional review board at Thomas Jefferson University. A prospectively collected database including all patients presenting to a regional spinal cord injury center ( 1 of 14 such centers designated by the National Institute on Disability and Rehabilitation Research) between January 2004 and June 2009 was retrospectively queried to identify those with a diagnosis of CCS. This diagnosis was based on the impressions of an orthopedic surgeon and neurosurgeon, both neurosurgery fellowship-trained spine surgeons, using the constellation of symptoms as well as the results of imaging studies. Furthermore, the diagnosis was agreed upon when the cases were presented at the morning spinal cord injury conference, which is attended by all available spine surgeons (orthopedic and neurosurgery). Patients treated nonsurgically were excluded from this study. AMS data collected throughout each patient's hospitalization were abstracted from the daily progress notes of the treating Physical Medicine \& Rehabilitation physicians. The data were collected in a standard manner, with the first collection taking place at the initial presentation and subsequently on morning rounds. The database and hospital records provided information regarding patient demographics, presenting neurological examination, imaging findings, medical comorbidities, timing and nature of the surgical procedures, length of stay (LOS) in the hospital, 
and LOS in the intensive care unit (ICU). Specifically, the presence of spinal cord edema was identified, based on the interpretations of the attending radiologist and a fellowship-trained spine surgeon. Patients were separated into those who underwent early surgery, within 1 day of presentation (early group), and those who underwent surgery on a delayed basis (delayed group). The decision for the patient to undergo early versus delayed surgery was made at the surgeon's discretion.

Differences between early and delayed groups were analyzed using the Student t-test for continuous variables and chi-square tests for categorical variables. Predictors of change in AMS between admission and 7 days after admission were identified using Spearman's correlation coefficients and backward stepwise multiple regression. Potential predictors of change in AMS included in the regression analysis were patient age, rectal tone on admission, admission AMS, presence of congenital stenosis, presence of spinal cord edema on MRI, and surgery within 1 day of admission. Significance was assumed for $\mathrm{p}<0.05$.

\section{Results}

Of 426 patients in the database, $80(18.8 \%)$ were diagnosed as having CCS, and 68 ultimately underwent decompressive surgery (Fig. 1). Nineteen (28\%) of 68 patients underwent surgery within 1 day of presentation (early group) while the remaining 49 patients $(72 \%)$ underwent surgery on a delayed basis (delayed group). The mean age of the early group was 52 years versus 59 years for the delayed group $(\mathrm{p}=0.049)$. Other characteristics were similar between groups including sex, proportion of patients with cord edema on MRI (44\% early vs 55\% delayed, $\mathrm{p}=0.47$ ), and proportion of patients with cervical fracture (26\% early vs $28 \%$ delayed, $\mathrm{p}=0.98$ ) (Table 1). Additionally, there was no difference in the number of patients who were treated in the ICU, allowing for continuous blood pressure management $(56 \%$ early vs $59 \%$ delayed, $\mathrm{p}=0.79)$.

Data on AMS at presentation and at 1 week after admission were available for $61(90 \%)$ of 68 patients. Of the 7 patients for whom 1-week AMS data were missing, 6 had been discharged to a rehabilitation center prior to 1 week after presentation; therefore, their neurological status was not captured in the database. One patient left the ICU prior to 1 week, and the medical record was insufficient to identify the post-ICU destination. No difference was noted between the surgical procedures performed on the early and delayed groups (Table 2). Patients in the early group presented with an AMS of 62.5 versus 70.0 for the delayed group, a nonsignificant difference $(p=0.36)$. At the 7 th day after admission, the early group had a mean AMS of 59.6, a nonsignificant loss of 2.9 points from the admission AMS $(p=0.39)$ while the delayed group had a mean AMS of 65.8 , representing a nonsignificant loss of 4.2 points ( $\mathrm{p}$ $=0.29$ ) (Table 3). The early and delayed groups thus did not have significant differences in AMS at the 7-day time point $(\mathrm{p}=0.34)$. In the early group, $50 \%$ of patients had an improvement in AMS score between admission and 7 days compared with $48 \%$ in the delayed group $(\mathrm{p}=0.94)$. The early group had a mean hospital LOS of 10.5 days and an ICU LOS of 3.4 days compared with a hospital LOS of 12.5 days $(\mathrm{p}=0.59)$ and ICU LOS of 3.4 days ( $\mathrm{p}$ $=0.84$ ) for the delayed group. No difference in mortality,
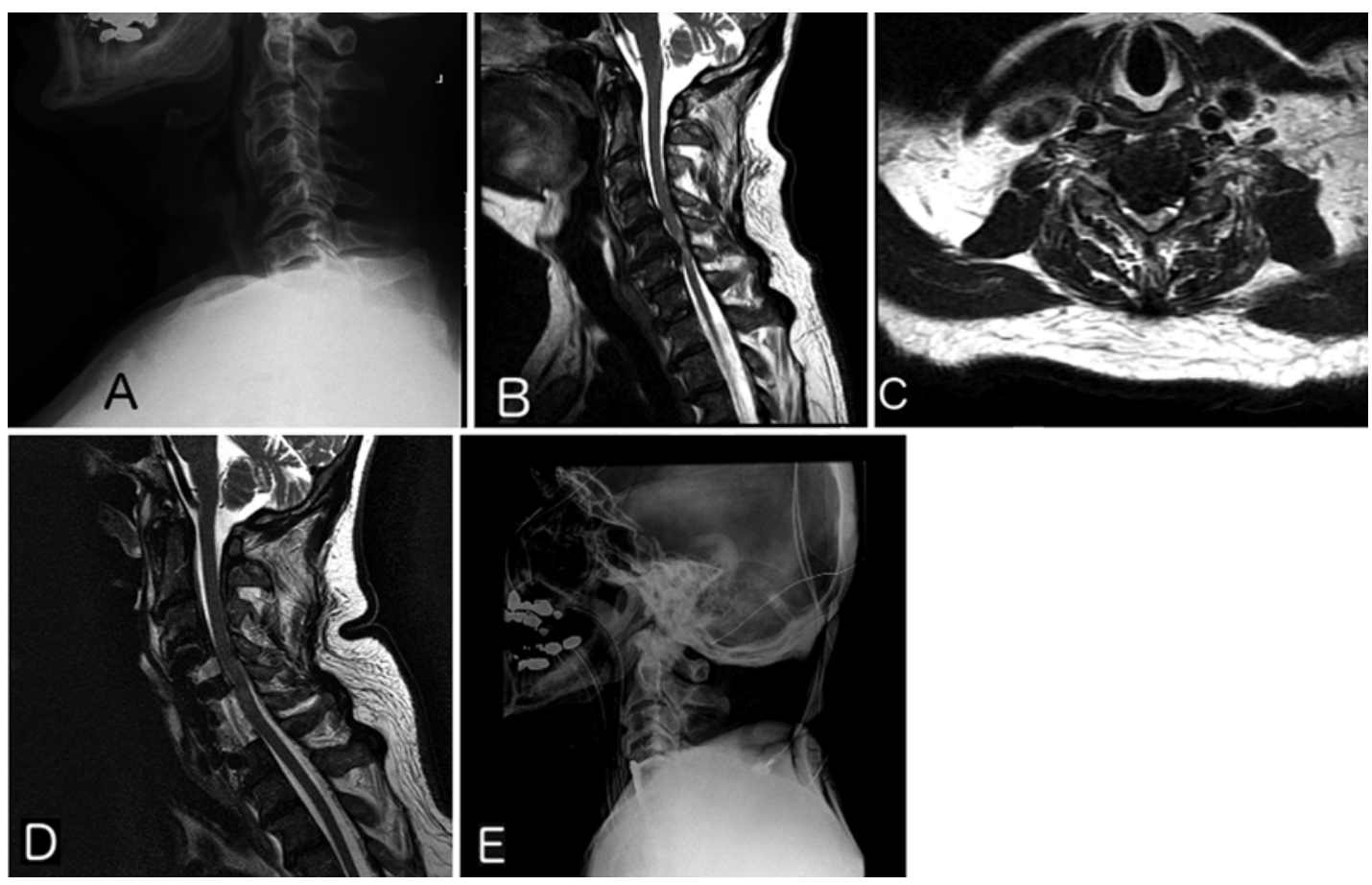

FIG. 1. Preoperative and postoperative images obtained in a 53-year-old man who presented with CCS and underwent surgical decompression 48 hours after injury. A: Preoperative lateral radiograph. B: Preoperative T2-weighted sagittal MR image. C: Preoperative axial T2-weighted image through C5-6. D: Postoperative sagittal T2-weighted MR image showing decompression of the spinal cord. E: Postoperative lateral radiograph. 
TABLE 1. Demographic and clinical characteristics of patients treated with early and delayed surgical decompression

\begin{tabular}{lccc}
\hline \multicolumn{1}{c}{ Characteristic } & $\begin{array}{c}\text { Early } \\
\text { Surgery }\end{array}$ & $\begin{array}{c}\text { Delayed } \\
\text { Surgery }\end{array}$ & p Value \\
\hline Age (yrs) & 52.1 & 59.2 & 0.049 \\
\hline Male (\%) & 63 & 72 & 0.56 \\
\hline Treated in ICU (\%) & 56 & 59 & 0.79 \\
\hline $\begin{array}{l}\text { Injuries that occurred on a weekend } \\
\text { (\%) }\end{array}$ & 26 & 35 & 0.58 \\
\hline Mean ISS score & 18.1 & 19.8 & 0.58 \\
\hline Mean GCS score & 15.0 & 14.9 & 0.48 \\
\hline Presence of cord edema (\%) & 44 & 55 & 0.47 \\
\hline Presence of fracture (\%) & 26 & 28 & 0.98 \\
\hline Mechanism of injury (\%) & & & \\
\hline Fall from height & 47 & 31 & 0.26 \\
\hline Fall from standing or sitting & 26 & 43 & 0.27 \\
\hline Motor vehicle accident & 16 & 20 & 0.99 \\
\hline Other & 11 & 6 & 0.61 \\
\hline
\end{tabular}

GCS = Glasgow Coma Scale; ISS = Injury Severity Score.

wound infection, wound hematoma, or venous thrombosis or embolism was identified between the groups (Table 3).

Analysis with Spearman's correlation coefficient demonstrated early surgery was not associated with a change in AMS between admission and Day 7 (correlation coefficient $-0.11, \mathrm{p}=0.44$ ) or AMS at the 7-day time point (correlation coefficient $0.05, \mathrm{p}=0.71$ ). Multiple linear regression demonstrated that rectal tone, admission AMS, presence of congenital stenosis, presence of a fracture, presence of spinal cord edema on MRI, and surgery within 1 day of admission were not predictive of a change in AMS score. The only significant predictor of change in AMS was patient age, which had a negative effect (regression coefficient $=-0.34, p=0.025$ ).

\section{Discussion}

Early treatment of patients with CCS remains controversial. Although some patients may present with unstable fractures that require immediate stabilization, such patients are the minority. ${ }^{8}$ In our study population, patients with cervical fractures were represented equally in both the early and delayed groups, accounting for $26 \%$ and $28 \%$ of patients in each groups, respectively.

By characterizing early outcomes in patients with CCS who undergo surgical decompression, we were unable to demonstrate any benefit of early operative intervention

TABLE 2. Surgical procedures performed in patients treated with early and delayed surgical decompression

\begin{tabular}{lccc}
\hline \multicolumn{1}{c}{ Procedure } & $\begin{array}{c}\text { Early } \\
\text { Surgery }\end{array}$ & $\begin{array}{c}\text { Delayed } \\
\text { Surgery }\end{array}$ & p Value \\
\hline Anterior decompression \& fusion & $32 \%$ & $27 \%$ & 0.77 \\
\hline Posterior decompression \& fusion & $53 \%$ & $53 \%$ & 0.99 \\
\hline $\begin{array}{c}\text { Combined anterior \& posterior } \\
\text { decompression \& fusion }\end{array}$ & $16 \%$ & $20 \%$ & 0.99 \\
\hline
\end{tabular}

TABLE 3. Early neurological recovery and complications in patients with CCS

\begin{tabular}{lccc}
\hline \multicolumn{1}{c}{ Variable } & $\begin{array}{c}\text { Early } \\
\text { Surgery }\end{array}$ & $\begin{array}{c}\text { Delayed } \\
\text { Surgery }\end{array}$ & p Value \\
\hline Initial AMS & 62.5 & 70 & 0.36 \\
\hline 7-day AMS & 59.6 & 65.8 & 0.34 \\
\hline Improved AMS at 1 wk (\%) & 50 & 48 & 0.94 \\
\hline Total LOS (days) & 10.5 & 12.5 & 0.59 \\
\hline LOS in ICU (days) & 3.4 & 3.4 & 0.84 \\
\hline Mortality (\%) & 0 & 0 & 0.99 \\
\hline Wound infection (\%) & 0 & 8 & 0.57 \\
\hline Postoperative hematoma (\%) & 0 & 4 & 0.99 \\
\hline DVT/PE (\%) & 5 & 4 & 0.99 \\
\hline Average no. of days on a ventilator & 1.7 & 2.0 & 0.87 \\
\hline >48 hrs on a ventilator (\%) & 11 & 10 & 0.99 \\
\hline
\end{tabular}

DVT = deep venous thrombosis; $\mathrm{PE}=$ pulmonary embolism.

with regard to AMS improvement or shortened LOS in the hospital or ICU. This finding was surprising given the significantly younger age of the early group compared with the delayed group (52 vs 59 years, $\mathrm{p}=0.049$ ). Not only has younger age been previously shown to be associated with improved outcomes in CCS, $, 3,11,15$ but regression analysis within our study demonstrated that younger age was the only significant predictor of improved outcome (regression coefficient $=-0.34, \mathrm{p}=0.025$ ).

Other than age, our analysis did not identify any other discriminator of early motor outcomes. This is consistent with previous literature, which did not find the presence of decreased rectal tone, cervical fracture, or congenital stenosis to have predictive value in terms of improvement in AMS.$^{1,4}$ Although AMS on admission ${ }^{10}$ and the presence of cord edema on MRI ${ }^{14}$ have previously been identified to have prognostic value, we found these variables to have no predictive value for early neurological recovery in our cohort. Further research with larger and more homogenous patient subpopulations may identify patient subgroups with differential benefit from early decompression.

The insignificant, average neurological decline witnessed in both groups was unexpected. Recent literature suggests that early decompression for both complete and incomplete cord injuries results in improved motor outcome scores. ${ }^{18}$ While documentation of the neurological recovery within the 1st week may not accurately describe the ultimate neurological recovery expected in this cohort, the lack of significant AMS recovery is valuable for clinicians treating these patients to define patient and physician expectations in this early recovery period. Prior to demonstrating obvious neurological recovery, these patients may experience a plateau in neurological function, even after decompressive surgery. Potential factors dictating the length of this period and ultimate recovery may still include previously described factors such as the degree of cord edema and presenting neurological injury, secondary injury, or the age of the patient.

Our study has limitations including those inherent to retrospective analysis. To a certain extent, this is mitigated by the prospective nature of the databases from which the 
data were obtained, but this was still not an a priori analysis. Also, follow-up AMS data were missing from $7 \mathrm{pa}-$ tients who were discharged before their 7-day postinjury AMS could be evaluated. In a study population of this size, it is possible but unlikely that such an omission would have had a significant effect on our observed results. Furthermore, while AMS is the best metric available for monitoring the neurological recovery after a spinal cord injury, because central cord preferentially affects the hands, it may not be as sensitive in detecting improvement in this diagnosis compared with other spinal cord injury patterns.

A second significant limitation of the study is the limited follow-up, and while undoubtedly long-term results are paramount, prior to this study, the literature did not allow for surgeons to give evidence-based counseling of the short-term expectations. In our institution, we found that after such a life-changing injury, patients were very anxious about what to expect not only in 1 year but also in 1 week. Furthermore, the knowledge that it is common to experience some worsening in the 1st week after surgery is particularly comforting to our patients when this does occur.

While our data are not necessarily representative of ultimate neurological recovery, they do lend insight to the early outcomes occurring in this unique patient population. Unlike previous studies that have focused on longerterm follow-up data, our study reported on earlier outcomes. While this limited our study from being directly compared with previous landmark papers, it provided the opportunity to better characterize patterns of recovery in finer detail. As our understanding of CCS pathophysiology continues to improve, the relevance of these findings may come into better realization to aid not only surgical planning but also forecasting postoperative recovery and rehabilitation. Further study into the risks and benefits of surgery and timing of intervention are necessary for this common and debilitating injury.

\section{Conclusions}

Early treatment of patients with CCS remains controversial; however, recent studies have reported improved long-term results with early surgical decompression. The current study finds no early neurological improvement after patients undergo early surgical decompression, so it is paramount for both surgeons and patients to understand that while some neurological recovery is expected after a CCS, the rehabilitation process will take months regardless of early surgical intervention.

\section{References}

1. Anderson DG, Sayadipour A, Limthongkul W, Martin ND, Vaccaro A, Harrop JS: Traumatic central cord syndrome: neurologic recovery after surgical management. Am J Orthop 41:E104-E108, 2012

2. Bosch A, Stauffer ES, Nickel VL: Incomplete traumatic quadriplegia. A ten-year review. JAMA 216:473-478, 1971

3. Dai L, Jia L: Central cord injury complicating acute cervical disc herniation in trauma. Spine (Phila Pa 1976) 25:331336,2000

4. Dvorak MF, Fisher CG, Hoekema J, Boyd M, Noonan V, Wing PC, et al: Factors predicting motor recovery and functional outcome after traumatic central cord syndrome: a long-term follow-up. Spine (Phila Pa 1976) 30:2303-2311, 2005 [Erratum in Spine (Phila Pa 1976) 31:1289]

5. Fawcett JW, Curt A, Steeves JD, Coleman WP, Tuszynski MH, Lammertse D, et al: Guidelines for the conduct of clinical trials for spinal cord injury as developed by the ICCP panel: spontaneous recovery after spinal cord injury and statistical power needed for therapeutic clinical trials. Spinal Cord 45:190-205, 2007

6. Harrop JS, Sharan A, Ratliff J: Central cord injury: pathophysiology, management, and outcomes. Spine J 6 (6 Suppl):198S-206S, 2006

7. Hendey GW, Wolfson AB, Mower WR, Hoffman JR: Spinal cord injury without radiographic abnormality: results of the National Emergency X-Radiography Utilization Study in blunt cervical trauma. J Trauma 53:1-4, 2002

8. Ishida Y, Tominaga T: Predictors of neurologic recovery in acute central cervical cord injury with only upper extremity impairment. Spine (Phila Pa 1976) 27:1652-1658, 2002

9. La Rosa G, Conti A, Cardali S, Cacciola F, Tomasello F: Does early decompression improve neurological outcome of spinal cord injured patients? Appraisal of the literature using a meta-analytical approach. Spinal Cord 42:503-512, 2004

10. Lenehan B, Fisher CG, Vaccaro A, Fehlings M, Aarabi B, Dvorak MF: The urgency of surgical decompression in acute central cord injuries with spondylosis and without instability. Spine (Phila Pa 1976) 35 (21 Suppl):S180-S186, 2010

11. Lenehan B, Street J, O'Toole P, Siddiqui A, Poynton A: Central cord syndrome in Ireland: the effect of age on clinical outcome. Eur Spine J 18:1458-1463, 2009

12. Marino RJ, Graves DE: Metric properties of the ASIA motor score: subscales improve correlation with functional activities. Arch Phys Med Rehabil 85:1804-1810, 2004

13. Merriam WF, Taylor TK, Ruff SJ, McPhail MJ: A reappraisal of acute traumatic central cord syndrome. J Bone Joint Surg Br 68:708-713, 1986

14. Miranda P, Gomez P, Alday R: Acute traumatic central cord syndrome: analysis of clinical and radiological correlations. J Neurosurg Sci 52:107-112, 2008

15. Penrod LE, Hegde SK, Ditunno JF Jr: Age effect on prognosis for functional recovery in acute, traumatic central cord syndrome. Arch Phys Med Rehabil 71:963-968, 1990

16. Schneider RC, Cherry G, Pantek H: The syndrome of acute central cervical spinal cord injury; with special reference to the mechanisms involved in hyperextension injuries of cervical spine. J Neurosurg 11:546-577, 1954

17. Stevens EA, Marsh R, Wilson JA, Sweasey TA, Branch CL $\mathrm{Jr}$, Powers AK: A review of surgical intervention in the setting of traumatic central cord syndrome. Spine J 10:874880,2010

18. Wilson JR, Singh A, Craven C, Verrier MC, Drew B, Ahn $\mathrm{H}$, et al: Early versus late surgery for traumatic spinal cord injury: the results of a prospective Canadian cohort study. Spinal Cord 50:840-843, 2012

\section{Author Contributions}

Conception and design: Kepler, Kong, Schroeder, Vaccaro, Anderson. Acquisition of data: Kepler, Kong, Hjelm, Sayadipour. Analysis and interpretation of data: all authors. Drafting the article: Kepler. Critically revising the article: all authors. Reviewed submitted version of manuscript: Kepler, Kong, Schroeder, Sayadipour, Vaccaro, Anderson. Statistical analysis: Kepler. Administrative/technical/material support: Kepler, Hjelm.

\section{Correspondence}

Christopher K. Kepler, Rothman Institute \& Thomas Jefferson University, 925 Chestnut St., 5th Fl., Philadelphia, PA 19107. email: chris.kepler@gmail.com. 